\title{
The problem of jitter and its correction for diagnostics at ASDEX Upgrade
}

\author{
Pascal de Marné, Albrecht Herrmann, and the ASDEX Upgrade Team \\ Max-Planck-Institut für Plasmaphysik, EURATOM Association, Garching, Germany
}

\begin{abstract}
Different optical diagnostics are operated at fusion devices like ASDEX Upgrade. One of them is thermography for contactless temperature measurements of surfaces in the vessel using infrared cameras. These temperatures can be used for the calculation of the heat flux on the monitored surfaces. But for the correct analysis of the recorded data stable measurement conditions regarding mechanical aspects like vibrations are needed. Since these conditions are often not fulfilled the measurements are affected by jitter. This paper describes a method used at ASDEX Upgrade for the correction of 1D and 2D thermographic data to compensate the jitter. It is based on a correlation analysis and also deals with jitter in the subpixel range. Examples are shown for the correction of temperature data and also for the influence on heat flux calculations with corrected and uncorrected temperatures. The limits of the proposed algorithm are also mentioned.
\end{abstract}

Keywords: thermography, jitter correction, ASDEX Upgrade

\section{Introduction}

Thermography is a mean for contactless and fast temperature measurements by measuring the temperature dependent emitted radiation with infrared cameras. At ASDEX Upgrade different thermographic diagnostics are used to monitor the surface temperature of structures in the inner-vessel. Two line cameras (called PPA and PPT) cooled by liquid nitrogen are installed vertically for a poloidal overview of the divertor. Up to three 2D cameras, each with a Stirling cooler, can be installed on different vacuum windows. For every 2D camera the used subarray of the FPA detector can be adjusted on a region of interest to increase the maximum frame rate. Two of the cameras are mounted on fixed windows while the third one uses an endoscope with two movable mirrors in front of the optic to vary the field of view.

Since the measured temperature evolution over time depends on the power load on the monitored surface, it is possible to calculate this load from the surface temperature data if the subsurface structure and all thermal characteristics are known. At ASDEX Upgrade these calculations are done with the THEODOR code [1]. For correct heat flux calculations it is necessary that always the same part of the monitored surface is imaged on a certain pixel. Otherwise temperatures from different positions will be used for the determination of the temperature evolution. In regions of homogenous temperatures this is no problem, but for regions with temperature gradients the use of this temperature traces for heat flux calculation can lead to erroneous results with much higher or lower values than really occurred in the experiment.

\section{The problem of jitter}

Even during normal operation the whole setup of the vacuum vessel and its surrounding diagnostics is not really stable resulting in vibrations of camera mountings, deflection mirrors or other parts of the experiment. Also the vibrations of the coolers of the $2 \mathrm{D}$ cameras can affect the FPA chip. Therefore the recorded data is affected by jitter. Figure 1 shows an image of the 1D temperature distribution over time for one of the divertor diagnostics. In the lower region a wavy behavior is visible which is not caused by a movement of the plasma but is due to vibrations. The observed displacements reach values around $10 \mathrm{~mm}$.

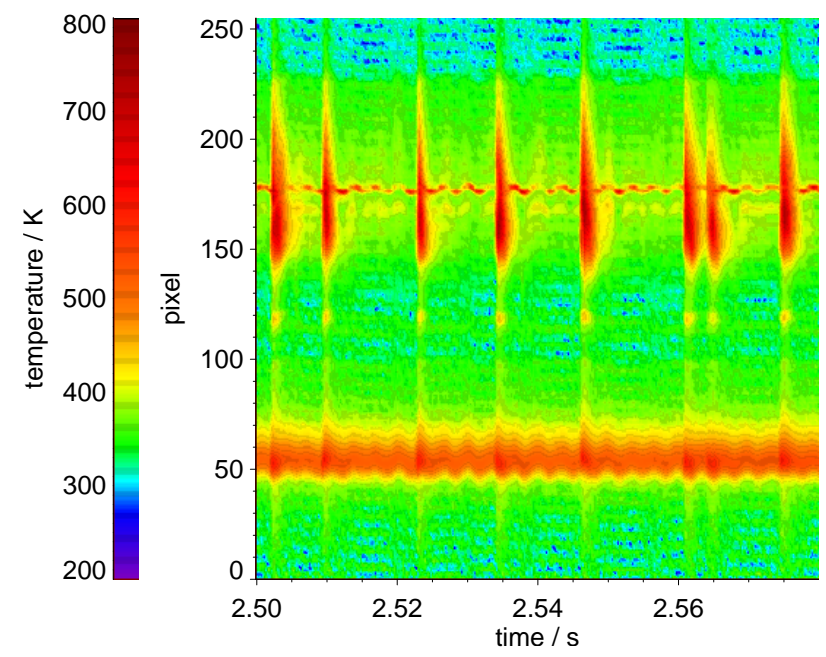

Fig.1 Measured temperature for the PPT diagnostic during shot \#21372. Resolution is about $2 \mathrm{~mm} /$ pixel.

Especially during disruptions there are much stronger forces affecting the device leading to more powerful vibrations and therefore the recorded data is strongly affected by jitter.

A similar problem exists for reciprocating probes if they are monitored by a camera. At ASDEX Upgrade the midplane manipulator can be equipped with different probes for measurements at the plasma edge and it is in the field of view of an IR camera. Also its strokes are not 
really jitter in terms of unwanted movements, they can be considered as jitter regarding temperature and heat flux analysis.

\section{Principle of correction}

The basic principle for the correction of $1 \mathrm{D}$ and $2 \mathrm{D}$ data is the same and consists of two major steps:

1. Automated determination of the amount of jitter between two consecutive frames or in regard to a fixed point.

2. Shifting of the processed data to compensate the jitter determined in step 1.

The determination in step 1 is done by a correlation analysis. Since the calculation of correlation values is quite robust against changes in the mean value it gives good results for the temperature data with its large changes.

The displacement between two consecutive frames is usually in the subpixel range (like a 'drift') for normal operation, much less then one whole pixel. Therefore the data is expanded in its dimensions by the factor of ten with interpolation before further processing. Afterwards the size is reduced again to its original values.

For the interpolation a (bi)linear interpolation is sufficient. The use of more complex interpolation algorithms like polynomial or spline interpolation does not yield in better results and only increases the needed computational time. The reducing is done by an averaging process.

\section{1. $1 \mathrm{D}$ case}

For both line cameras horizontal gaps between two divertor tiles are imaged on the CCD. Since these gaps are temperature independent they can be used as a fix point for the correlation analysis. Instead of using a part of the measured data as a reference pattern a simple mathematical parabola is used. In figure 2 the parabola is shown compared to a typical time slice of real data, where also the gap is visible.

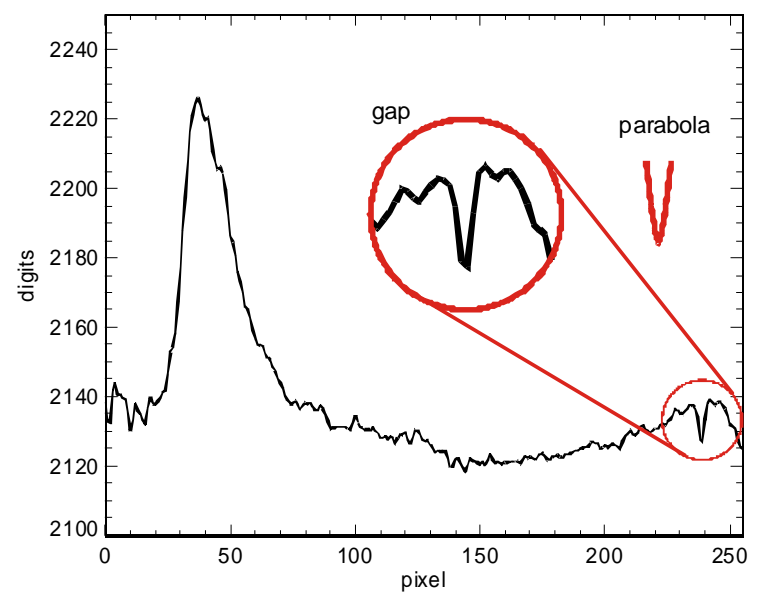

Fig.2 Example temperature for one time point with the gap used as fix point compared to the parabola used for the correlation analysis.
The parabola is shifted over the cutout of the data where the gap is expected. For every shifting position the correlation between data and parabola is calculated and the number of the subpixel with the maximum correlation value is stored in a vector of shifting values.

Before performing the shifting of the data set a plausibility check is done for the vector. Only a small displacement between two consecutive frames is expected and therefore the vector should have a smooth and steady shape without isolated extreme values. Thus the vector itself is corrected by eliminating outliers with a median filter.

\section{2. $2 \mathrm{D}$ case}

For the 2D data it is not possible to use one fixed pattern for the correlation analysis since the field of view varies with the used subarray and with the mirror positions in case of the camera with the endoscope. It also depends on the parameters of a discharge where the temperature distribution allows an identification of useable parts of the monitored structure. Therefore the user has to select an adequate pattern for the correlation analysis. This pattern has to be part of the structure and must not be a result of a temperature distribution (although its contrast may be enhanced due to temperature changes) because a temperature distribution can change due to moving of the plasma.

In addition to the drift like jitter as for the $1 \mathrm{D}$ data the 2D data is also sometimes affected by 'jumps', which means a large deviation of several whole pixels between two consecutive frames. Therefore the process of jitter determination is split into two steps to save computational time, the rough and the fine determination. At first the jump detection is done by correlating the processed frame with the previous processed frame. Both frames are neither shifted nor expanded for this calculation. If the correlation value falls below a previously defined threshold it is assumed that a jump had occurred between these two frames and the rough determination is executed. Otherwise it is skipped and the fine determination is applied [2]. For the rough determination the reference pattern is shifted over the whole processed frame. Also in this step neither pattern nor frame are expanded. The position of the pattern with the maximum correlation value is forwarded to the following fine determination as starting position.

The fine determination deals with the drift. It uses the expanded reference pattern with the expanded data set. The search for the maximum correlation is done in iterative steps. The standard starting position for the reference pattern is the jitter value determined for the previous frame. Only if a jump had been detected the result of the rough determination is used as start point. The correlation is calculated for a 3x3 neighborhood around the starting pixel. If the maximum value for these nine positions refers to the centre pixel the fine determination is done. Otherwise a new $3 \times 3$ neighborhood is processed with its new centre at the position with the maximum correlation in the last step. This is repeated until either the maximum correlation is 
calculated for the centre pixel or a predefined distance to the starting position is exceeded. In the second case an error message is generated and the correction is aborted. Otherwise the distance between the starting position and the current centre pixel is the determined jitter. The processed frame is shifted by this value and saved before the next frame is processed.

\section{Examples}

Figure 3 shows the jitter corrected data of figure 1 . The wavy form in the lower region has vanished and a much smoother temperature evolution is visible only disturbed by ELMs.

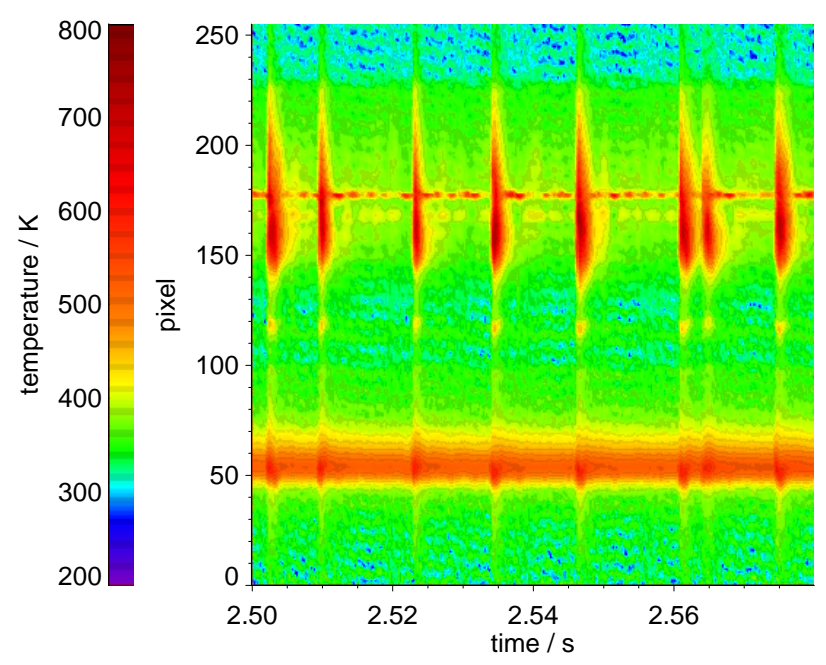

Fig.3 Temperature data from figure 1 after applying jitter correction.

The maximum temperature of the region between pixel 150 and 174 with and without correction is shown in figure 4. Only small differences are found as expected.

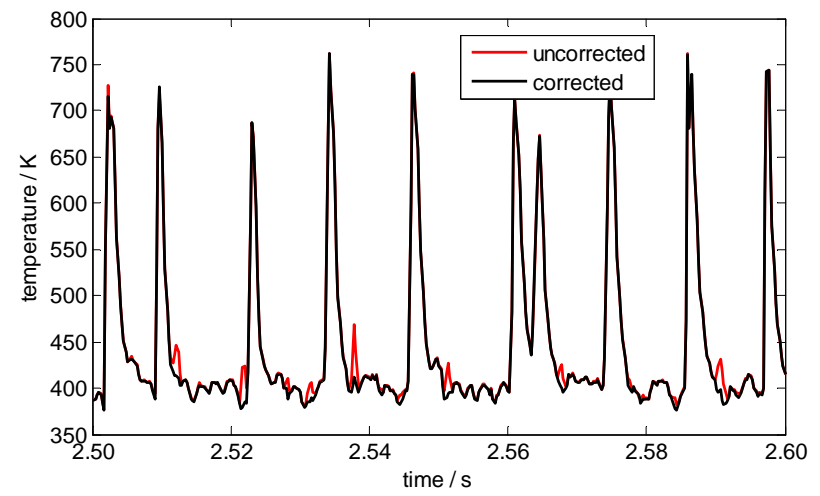

Fig.4 Maximum temperature for the region between pixel 150 and 174.

But for the calculated heat flux significant differences are found as shown in figure 5. The maxima determination analyzes a region of pixels and is therefore insensitive against jitter while the calculation of the heat flux happens pixel-wise over time. Thus the heat flux is influenced by the jitter and therefore also its maxima.

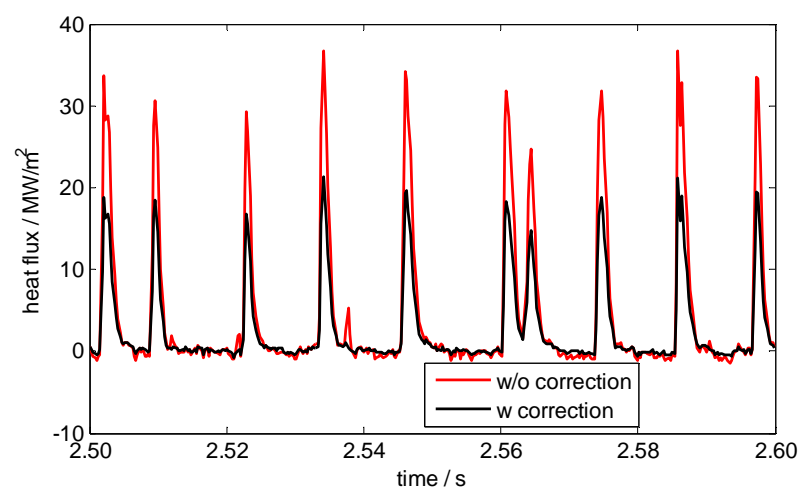

Fig.5 Maximum heat flux for the region between pixel 150 and 174.

Without correction the heat flux reaches up to twice as high values compared to corrected values. As compensation the values for the heat flux between two ELMs are much lower and reach high negative values. This can be seen in figure 6 showing the heat flux for a single pixel in the analyzed region. Both temperature and heat flux evolution are very shaky.

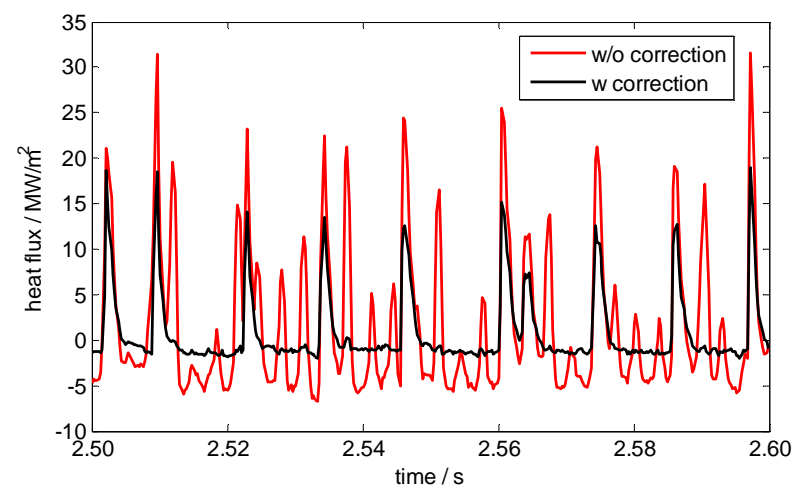

Fig.6 Heat flux evolution at pixel 175.

In a laboratory experiment a CFC sample was heated by a short laser pulse. The temperature was measured with a 2D camera with insufficient decoupling between FPA and cooler resulting in a wobbling of the chip in respect to the observed sample and therefore a jitter in the recorded data. Figure 7 shows the maximum heat flux calculated from the data and figure 8 the minimum heat flux. With uncorrected temperatures much too high values and an oscillation are occurring while also high negative values can bee seen in the minimum plot.

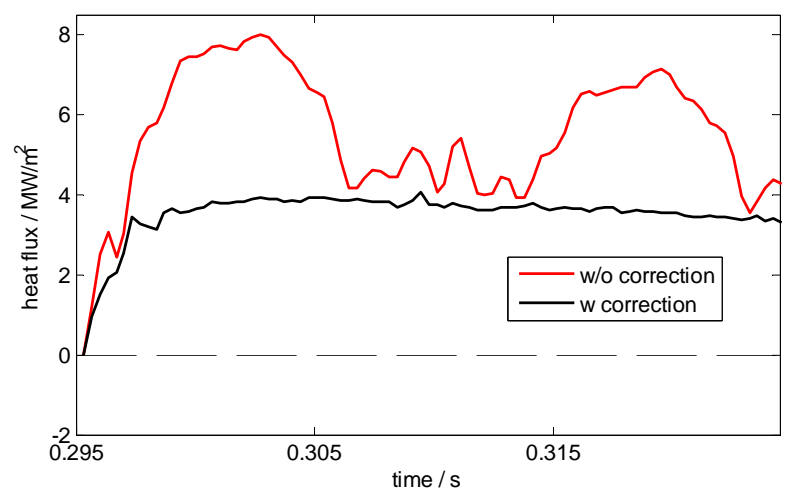

Fig.7 Maximum heat flux for CFC sample after laser pulse. 


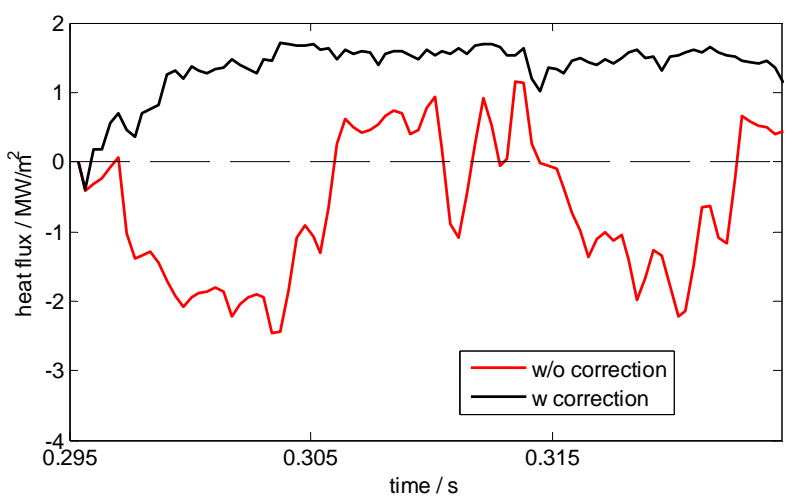

Fig.8 Minimum heat flux for CFC sample.

Of course heating with a laser cannot lead to a negative heat flux. With the corrected temperatures the heat flux result looks like expected for a laser pulse.

As a second example for a $2 \mathrm{D}$ correction a probe movement is compensated. Figure 9 shows the signal of the position signal of the ASDEX Upgrade manipulator during one stroke while the probe head was in the field of view for the camera.

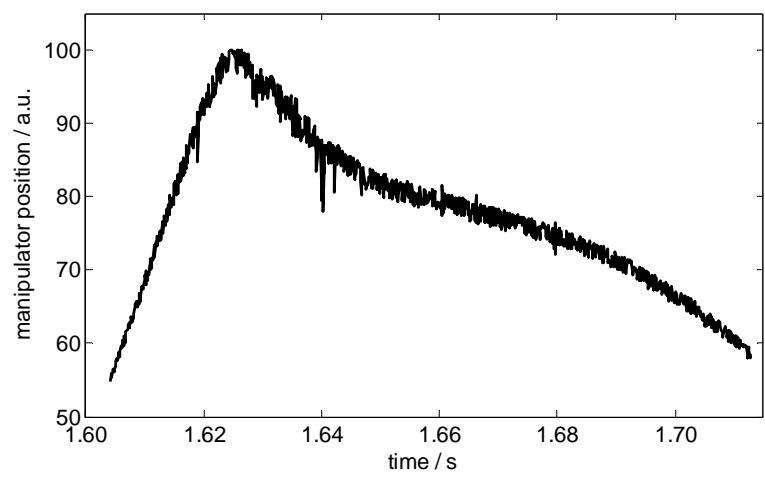

Fig.9 Position signal from the manipulator probe.

In figure 10 the determined jitter in both pixel directions is shown. The reference pattern was taken from the frame at $1.635 \mathrm{~s}$ and therefore for this frame the jitter is 0 in both directions and the other values are relative to this frame.

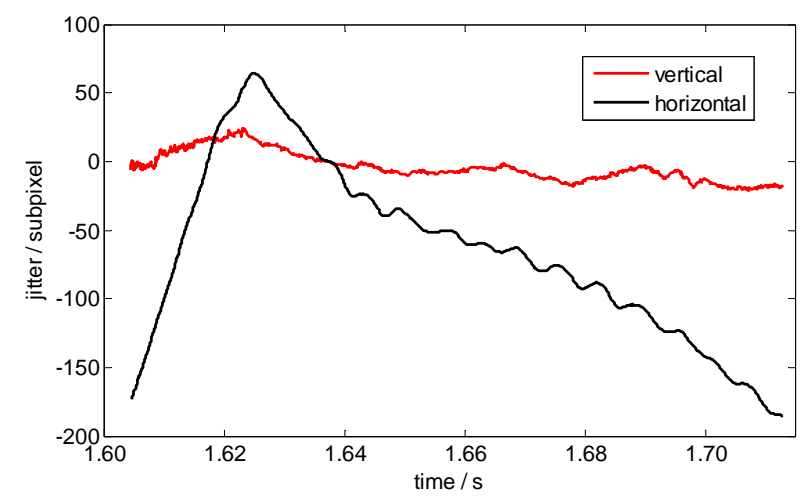

Fig.10 Determined jitter for the probe head. Because the head moves slightly tilted into the field of view the jitter is visible in both pixel directions.

The position signal and the jitter have a good agreement. Only at the end of the stroke when the probe leaves the field of view of the camera the deviation gets bigger because there are not enough pixels left for a convincing correlation.

\section{Limits}

Due to the high computational effort the method is not capable for realtime jitter correction. At the moment that is no drawback for the thermography at ASDEX Upgrade because also the data saving and the conversion from raw data to temperatures are done after a discharge and are not available during the shot.

The correction of the 1D data can be run automatically, but for the $2 \mathrm{D}$ data this is not possible. For each discharge the reference pattern used for the correlation analysis has to be defined separately by the user depending on the recorded data set. Sometimes the pattern has to be exchanged one or more times during the processing of the data set for a sufficient determination of the jitter. In cases with very small subframes it is often not possible to define a useful pattern. The need for a new reference pattern according to the measured data is another reason why a realtime application for the 2D correction is not feasible at the moment.

The data expanding by interpolation and the reducing after the correction to deal with the subpixel jitter has the same effect like using a smoothing filter on the data. Even for unshifted frames single pixels with extreme values will be lost after the processing of the data. This modification of unshifted frames could be avoided by replacing the averaging with simple resampling. But resampling gives only better results for frames which were not shifted or shifted by whole pixels. For other frames new errors can be created regarding the temporal evolution.

The described method can correct jitter based on translation movements. For the data recorded with the camera with the endoscope also a rotational jitter can occur which would need a more sophisticated algorithm.

\section{Conclusion}

The proposed method for the correction of jitter affected data has shown to give good results for both 1D and 2D data. Artefacts in calculated heat fluxes are significantly reduced with corrected temperature input data.

Although only used for thermographic data until now the proposed method is basically usable for other diagnostics relying on a group of lines of sight with a defined assembly in relation to each other. The only requirement is a usable reference pattern which is as independent as possible regarding the behavior of the experiment.

\section{References}

[1] A. Herrmann et al., Plasma Phys. Controlled Fusion 37, 17 (1995)

[2] H. Greuner et al., Phys. Scr. T138, 014063 (2009) 